\title{
Department of Mathematics
}

\section{A New High Order Energy and Enstrophy} Conserving Arakawa-like Jacobian Differential Operator

Chiara Sorgentone, Cristina La Cognata and Jan Nordström

$$
\text { LiTH-MAT-R--2015/05--SE }
$$

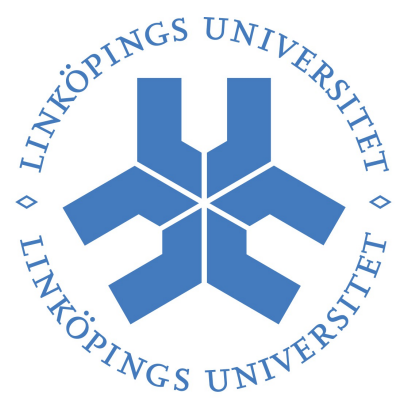

\section{Linköpings universitet}

TEKNISKA HÖGSKOLAN 
Department of Mathematics

Linköping University

S-581 83 Linköping, Sweden. 


\title{
A New High Order Energy and Enstrophy Conserving Arakawa-like Jacobian Differential Operator
}

\author{
Chiara Sorgentone $^{\mathrm{a}}$, Cristina La Cognata ${ }^{\mathrm{b}}$, Jan Nordström ${ }^{\mathrm{c}}$ \\ ${ }^{a}$ Department of Mathematics, Royal Institute of Technology (KTH), SE-100 44 \\ Stockholm, Sweden (sorgento@kth.se). \\ ${ }^{b}$ Department of Mathematics, Computational Mathematics, Linköping University, \\ SE-581 83 Linköping, Sweden (cristina.la.cognata@liu.se). \\ ${ }^{c}$ Department of Mathematics, Computational Mathematics, Linköping University, \\ SE-581 83 Linköping, Sweden (jan.nordstrom@liu.se).
}

\begin{abstract}
A new high order Arakawa-like method for the incompressible vorticity equation in two-dimensions has been developed. Mimetic properties such as skewsymmetry, energy and enstrophy conservations for the semi-discretization are proved for periodic problems using arbitrary high order summation-by-parts operators. Numerical simulations corroborate the theoretical findings.

Keywords: non-linear problems, summation-by-parts operators, Jacobian, mimetic schemes, high-order schemes, stability, finite difference
\end{abstract}

\section{Introduction}

The numerical discretization issue for non-linear problems has been studied for a long time. Among the many difficulties, the false transfer of energy between different scales and the consequent non-linear computational instability are arguably the hardest to encounter. How this problem can harm long-term numerical simulations and applications has first been shown by Phillips in [23] and afterwords by Durran in [9]. The non-linear numerical instability was traced back to the inappropriate treatment of the non-linear terms.

In order to overcome this problem, different solutions have been proposed: adding a smoothing term to control the non-physical energy growth [23],[15],[11], using higher order schemes to raise the accuracy [25], and using mimetic schemes to mimic physical properties [1],[14],[20],[2] are among 
the most common ones. Arakawa, in [3], proposed a particular second-order mimetic finite-difference Jacobian operator and showed the superiority of his solution with respect to other non-mimetic schemes [4]. His solution have been widely used [25],[12], studied [18],[8], and generalized [7],[24],[19].

The aim of this paper is to blend high order accuracy with mimetic properties and re-formulate Arakawa's Jacobian using summation-by-parts (SBP) operators $([6],[13],[27],[26])$. The proficiency of this technique lies in the simulation of the integration by parts property and maintain the analytical conservation properties in the discrete setting. All mimetic properties are included in the special matrix structure and do not depend on a specific discretization order. In Arakawa's scheme, for example, the mimetic properties are proved only for a second order scheme. By using SBP operators we aim for arbitrary high order approximations.

The paper is divided as follows: in Section 2, we derive the two-dimensional incompressible vorticity equation in terms of stream-function and vorticity and show well-posedness for a periodic problem. In Section 3 we analyze the analytical properties of the Jacobian operator which guarantee energy and enstrophy conservations for periodic problems. Summation-by-parts discretization and the corresponding conservation properties are constructed and proved in Section 4 by exploiting the SBP properties. Section 5 is dedicated to numerical computations and discussion of their results. Finally, we draw conclusions in Section 6.

\section{The incompressible vorticity equation}

Consider the Euler equations for an incompressible inviscid fluid in $\Re^{3}$

$$
\begin{aligned}
\frac{\partial \mathbf{v}}{\partial t}+\mathbf{v} \cdot \nabla \mathbf{v} & =-\nabla p \\
\nabla \cdot \mathbf{v} & =0
\end{aligned}
$$

where $\mathbf{v}=(u, v, w)$ is the velocity field in a Cartesian frame of coordinates $(x, y, z)$. The vorticity vector $\omega$ is defined as the curl of the velocity field $\mathbf{v}$, i.e.,

$$
\omega=\nabla \times \mathbf{v}=\left(\frac{\partial w}{\partial y}-\frac{\partial v}{\partial z}, \frac{\partial u}{\partial z}-\frac{\partial w}{\partial x}, \frac{\partial v}{\partial x}-\frac{\partial u}{\partial y}\right)
$$

In order to derive the vorticity equation we recall the identity

$$
\mathbf{v} \cdot \nabla \mathbf{v}=\omega \times \mathbf{v}+\nabla \frac{\left(|\mathbf{v}|^{2}\right)}{2}
$$


(for details see $[22],[16]$ ). By taking the curl of the momentum equation in (1), the incompressibility relation and (3) lead to

$$
\frac{\partial \omega}{\partial t}+\mathbf{v} \cdot \nabla \omega-\omega \cdot \nabla \mathbf{v}=0
$$

A two-dimensional flow can be described by a three-dimensional velocity field with the vertical component $w$ identically zero and the vorticity $\omega=(0,0, \zeta)$, where $\zeta=(\partial v / \partial x-\partial u / \partial y)$. In this setting, $\omega \perp \nabla \mathbf{v}$ and (4) becomes the vorticity equation for an incompressible flow on a general two-dimensional spatial domain. For completeness we restate (1) as:

$$
\begin{aligned}
\frac{\partial \zeta}{\partial t}+\mathbf{v} \cdot \nabla \zeta & =0 \\
\nabla \cdot \mathbf{v} & =0
\end{aligned}
$$

\section{Analytical properties of the Jacobian for periodic problems}

The divergence relation (6) implies (see [17],[5]) the existence of a primitive function $\psi$ with the exact differential form $d \psi=-u d y+v d x$. Therefore, zero divergence also implies that the velocity field can be written as

$$
(u, v)=\left(-\frac{\partial \psi}{\partial y}, \frac{\partial \psi}{\partial x}\right)
$$

The function $\psi$ in (7) is the so called stream function. By taking the curl of (7), it follows that the relation between the stream function $\psi$ and the vorticity $\zeta$ is $\zeta=\Delta \psi$. In this setting (5) can be rewritten as

$$
\frac{\partial \zeta}{\partial t}+J(\psi, \zeta)=0
$$

The advection term in (8) is expressed by the Jacobian differential operator $J(\cdot, \cdot)$, which is defined as

$$
J(a, b)=\frac{\partial a}{\partial x} \frac{\partial b}{\partial y}-\frac{\partial a}{\partial y} \frac{\partial b}{\partial x}
$$

By definition, the Jacobian operator satisfies naturally the skew-symmetric property:

$$
J(a, b)=-J(b, a)
$$


Moreover, if we consider periodic functions $a$ and $b$ on the spatial domain $D$, the following integral constraints on (9) hold:

$$
\int_{D} J(a, b) d x d y=\int_{D} a J(a, b) d x d y=\int_{D} b J(a, b) d x d y=0 .
$$

These last properties follow directly from integration by parts using periodic boundary conditions.

\subsection{Conservation properties}

We start with two definitions.

Definition 1. The mean kinetic energy for the equation (8) is defined as:

$$
K=\frac{1}{2} \overline{(\nabla \psi)^{2}}
$$

where $\frac{1}{|D|} \bar{f}=\int_{D} f d x d y$.

Definition 2. The enstrophy (mean square vorticity) for the equation (8) is defined as:

$$
G=\frac{1}{2} \overline{\zeta^{2}}
$$

In the following theorem we state the continuous conservation laws that are the main focus in this paper.

Theorem 1. Consider a periodic stream function with periodic related vorticity function. The vorticity equation (8) conserves the mean kinetic energy (12) and the enstrophy (13).

Proof. We multiply (8) by $\zeta$ and compute the mean over the domain $D$. This leads to

$$
\overline{\zeta \frac{\partial \zeta}{\partial t}}=\overline{\frac{1}{2} \frac{\partial \zeta^{2}}{\partial t}}=-\overline{\zeta J(\psi, \zeta)}=0
$$

where in the last equality we used the integral property (11). The result (14) shows that the formulation (8) conserves the enstrophy.

Next we consider conservation of mean kinetic energy; we multiply (8) by $\psi$ and compute the mean over the domain D. By using property (11) and integration by parts we get:

$$
\overline{\frac{\partial}{\partial t}\left(\frac{1}{2} \nabla \psi\right)^{2}}=\overline{\nabla \psi \frac{\partial \nabla \psi}{\partial t}}=-\overline{\psi \frac{\partial(\Delta \psi)}{\partial t}}=-\overline{\psi \frac{\partial \zeta}{\partial t}}=\overline{\psi J(\psi, \zeta)}=0 .
$$

The relation (15) show that (8) conserves mean kinetic energy. 
As a direct consequence of the enstrophy conservation, the periodic initial value problem associated with the vorticity equation is well-posed.

Corollary 1. The Cauchy problem given by

$$
\begin{aligned}
\frac{\partial \zeta}{\partial t}+J(\psi, \zeta) & =0, & & (x, y) \in D, t \geq 0, \\
\zeta(x, y, 0) & =f(x, y), & & (x, y) \in D,
\end{aligned}
$$

where $\zeta$ and $\psi$ are periodic functions on $D$, is well-posed.

Proof. The procedure used in (14) leads to

$$
\frac{\partial}{\partial t}\|\zeta\|_{D}^{2}=0
$$

where $\|\cdot\|$ indicates the standard $L^{2}$ norm. Integration with respect to the time leads to

$$
\|\zeta\|_{D}^{2}=\|f\|_{D}^{2}, \quad(x, y) \in D, t \geq 0 .
$$

The bound of the solution in terms of initial data guarantees well-posedness, see [10] for more details.

\subsection{Operator splitting}

Following the splitting technique presented in [21], we formulate a new Jacobian expression as a linear combination of three equivalent differential operators. These are:

$$
\begin{aligned}
J_{1}(\psi, \zeta) & =\left(\frac{\partial \psi}{\partial x} \frac{\partial \zeta}{\partial y}-\frac{\partial \psi}{\partial y} \frac{\partial \zeta}{\partial x}\right) \\
J_{2}(\psi, \zeta) & =\left(\frac{\partial}{\partial x}\left(\psi \frac{\partial \zeta}{\partial y}\right)-\frac{\partial}{\partial y}\left(\psi \frac{\partial \zeta}{\partial x}\right)\right) \\
J_{3}(\psi, \zeta) & =\left(\frac{\partial}{\partial y}\left(\zeta \frac{\partial \psi}{\partial x}\right)-\frac{\partial}{\partial x}\left(\zeta \frac{\partial \psi}{\partial y}\right)\right) .
\end{aligned}
$$

The general linear combination

$$
J(\psi, \zeta)=\alpha J_{1}+\beta J_{2}+\gamma J_{3}
$$

is a new operator equivalent to (9) for any set of coefficients $\alpha, \beta$ and $\gamma$, provided that $\alpha+\beta+\gamma=1$. It is equivalent in the sense that (19) is skewsymmetric and satisfies the integral constraints (14)-(15). 
In the remaining part of the paper, we will derive high order finite difference approximation of (19) which mimics identically the three properties in the discrete case. The result will be a high order accurate generalization of the second order Arakawa's Jacobian scheme in [3].

\section{Summation-by-parts operators for periodic problems}

To define an SBP semi-discretization of the spatial derivatives, we introduce the computational grid, $x_{i}=i h_{x}, i \in 0,1,2, \ldots, N$ and $y_{j}=j h_{y}$, $j \in 0,1,2, \ldots, M$ where $h_{x}$ and $h_{y}>0$ are the spatial steps in the two dimensions. The partial derivatives are approximated by

$$
\frac{\partial f}{\partial x} \approx\left(P_{x}^{-1} Q_{x} \otimes I_{y}\right) \mathbf{f} \quad \text { and } \quad \frac{\partial f}{\partial y} \approx\left(I_{x} \otimes P_{y}^{-1} Q_{y}\right) \mathbf{f}
$$

where $\mathbf{f}=\left(f_{11}, \ldots, f_{1 M}, f_{21} \ldots, f_{2 M}, \ldots, f_{N 1}, \ldots, f_{N M}\right)^{T}$ is a vector of dimension $N M$ and $\otimes$ is the Kronecker product of two matrices defined as

$$
A \otimes B=\left[\begin{array}{ccc}
a_{11} B & \ldots & a_{1 n} B \\
\vdots & & \vdots \\
a_{m 1} B & \ldots & a_{m n} B
\end{array}\right] .
$$

$P_{x, y}$ are diagonal positive definite matrices such that $\left(P_{x} \otimes P_{y}\right)$ define a twodimensional $L^{2}$ norm by $\|\mathbf{f}\|_{\left(P_{x} \otimes P_{y}\right)}^{2}=\mathbf{f}^{T}\left(P_{x} \otimes P_{y}\right) \mathbf{f}$. The matrices $Q_{x, y}$ are periodic operators satisfying $Q_{x, y}+Q_{x, y}^{T}=0$.

In order to define the discrete non-linear operator $J(\psi, \zeta)$ in $(19)$, we need to define an analogous of the product between two functions. For any vector $\mathbf{a}=\left(a_{1}, \ldots, a_{N}\right)$, let the matrix $\operatorname{diag}(\mathbf{a})$ be

$$
\operatorname{diag}(\mathbf{a})=\left[\begin{array}{ccccc}
a_{1} & 0 & 0 & \ldots & 0 \\
0 & a_{2} & 0 & \ldots & 0 \\
\vdots & & \vdots & & \vdots \\
0 & 0 & \ldots & 0 & a_{N}
\end{array}\right]
$$

and let the vector $\mathbf{1}=(1, \cdots, 1)^{T}$ be of the same length as $\mathbf{a}$. Then we have

$$
\operatorname{diag}(\mathbf{a}) \mathbf{1}=\mathbf{a} \text { and } \mathbf{1}^{T} \operatorname{diag}(\mathbf{a})=\mathbf{a}^{T},
$$

with properties:

$$
\operatorname{Ddiag}(\mathbf{a})=\operatorname{diag}(D \mathbf{a})
$$




$$
\mathbf{a}^{T} B=\left(B^{T} \mathbf{a}\right)^{T}=\mathbf{1}^{T} \operatorname{diag}\left(B^{T} \mathbf{a}\right),
$$

for any diagonal matrix $\mathrm{D}$, and for any vector a of length $N$ and $N \times N$ matrix $B$. Equation (24) follows from (22).

The definition (22) is used as follows to define the vectors:

$$
\begin{aligned}
& D_{x} \mathbf{f}=\left(P_{x}^{-1} Q_{x} \otimes I_{y}\right) \mathbf{f}=\operatorname{diag}\left(P_{x}^{-1} Q_{x} \otimes I_{y} \mathbf{f}\right) \mathbf{1}, \\
& D_{y} \mathbf{f}=\left(I_{x} \otimes P_{y}^{-1} Q_{y}\right) \mathbf{f}=\operatorname{diag}\left(I_{x} \otimes P_{y}^{-1} Q_{y} \mathbf{f}\right) \mathbf{1} .
\end{aligned}
$$

Now we can define the corresponding SBP discretization of the analytical Jacobian (19) by adopting the previous definitions for each term in (18). From (25), the discrete analogous of the differential operators in (18) are vectors of size NM, defined as

$$
\begin{aligned}
\mathbf{J}_{1}(\boldsymbol{\psi}, \boldsymbol{\zeta}) & =\left\{\left[\operatorname{diag}\left(P_{x}^{-1} Q_{x} \otimes I_{y} \boldsymbol{\psi}\right) \operatorname{diag}\left(I_{x} \otimes P_{y}^{-1} Q_{y} \boldsymbol{\zeta}\right)\right] \mathbf{1}\right. \\
& \left.-\left[\operatorname{diag}\left(I_{x} \otimes P_{y}^{-1} Q_{y} \boldsymbol{\psi}\right) \operatorname{diag}\left(P_{x}^{-1} Q_{x} \otimes I_{y} \boldsymbol{\zeta}\right)\right] \mathbf{1}\right\}, \\
\mathbf{J}_{2}(\boldsymbol{\psi}, \boldsymbol{\zeta}) & =\left\{\left(P_{x}^{-1} Q_{x} \otimes I_{y}\right)\left[\operatorname{diag}(\boldsymbol{\psi}) \operatorname{diag}\left(I_{x} \otimes P_{y}^{-1} Q_{y} \boldsymbol{\zeta}\right)\right] \mathbf{1}\right. \\
& \left.-\left(I_{x} \otimes P_{y}^{-1} Q_{y}\right)\left[\operatorname{diag}(\boldsymbol{\psi}) \operatorname{diag}\left(P_{x}^{-1} Q_{x} \otimes I_{y} \boldsymbol{\zeta}\right)\right] \mathbf{1}\right\}, \\
\mathbf{J}_{3}(\boldsymbol{\psi}, \boldsymbol{\zeta}) & =\left\{\left(I_{x} \otimes P_{y}^{-1} Q_{y}\right)\left[\operatorname{diag}(\boldsymbol{\zeta}) \operatorname{diag}\left(P_{x}^{-1} Q_{x} \otimes I_{y} \boldsymbol{\psi}\right)\right] \mathbf{1}\right. \\
- & \left.\left(P_{x}^{-1} Q_{x} \otimes I_{y}\right)\left[\operatorname{diag}(\boldsymbol{\zeta}) \operatorname{diag}\left(I_{x} \otimes P_{y}^{-1} Q_{y} \boldsymbol{\psi}\right)\right] \mathbf{1}\right\},
\end{aligned}
$$

where $\zeta$ and $\boldsymbol{\psi}$ are also vectors of size NM. Then, the discretization of (19) in SBP formulation may be obtain by the linear combinations of $\mathbf{J}_{1}, \mathbf{J}_{2}$ and $\mathbf{J}_{3}$, given by

$$
\mathbf{J}=\alpha \mathbf{J}_{1}+\beta \mathbf{J}_{2}+\gamma \mathbf{J}_{3},
$$

where the real constants $\alpha, \beta$ and $\gamma$ satisfy

$$
\alpha+\beta+\gamma=1 .
$$

The following theorem proves that the analytical properties (10) and (14)(15) are reproduced exactly by (29) for one unique choice of the coefficients in (30).

Theorem 2. The linear combination (29) is skew-symmetric, conserves enstrophy and conserves kinetic energy if and only if

$$
\alpha=\beta=\gamma=\frac{1}{3} .
$$


This choice gives the discrete Jacobian of arbitrary order of accuracy

$$
\boldsymbol{J}^{*}=\frac{1}{3} \boldsymbol{J}_{1}+\frac{1}{3} \boldsymbol{J}_{2}+\frac{1}{3} \boldsymbol{J}_{3}
$$

Proof. The proof is divided in three parts:

1. Conservation of enstrophy

Consider the semi-discretization

$$
\frac{\partial \boldsymbol{\zeta}}{\partial t}+\mathbf{J}(\boldsymbol{\psi}, \boldsymbol{\zeta})=0
$$

By multiplying (33) with $\zeta^{T}\left(P_{x} \otimes P_{y}\right)$ we have

$$
\begin{array}{r}
\boldsymbol{\zeta}^{T}\left(P_{x} \otimes P_{y}\right) \frac{\partial \boldsymbol{\zeta}}{\partial t}=\frac{1}{2} \frac{\partial}{\partial t}\|\boldsymbol{\zeta}\|_{\left(P_{x} \otimes P_{y}\right)}^{2} \\
=-\alpha \boldsymbol{\zeta}^{T}\left(P_{x} \otimes P_{y}\right)\left\{\left[\operatorname{diag}\left(P_{x}^{-1} Q_{x} \otimes I_{y} \boldsymbol{\psi}\right) \operatorname{diag}\left(I_{x} \otimes P_{y}^{-1} Q_{y} \boldsymbol{\zeta}\right)\right] \mathbf{1}\right. \\
\left.-\left[\operatorname{diag}\left(I_{x} \otimes P_{y}^{-1} Q_{y} \boldsymbol{\psi}\right) \operatorname{diag}\left(P_{x}^{-1} Q_{x} \otimes I_{y} \boldsymbol{\zeta}\right)\right] \mathbf{1}\right\} \\
-\beta \boldsymbol{\zeta}^{T}\left(P_{x} \otimes P_{y}\right)\left\{\left(P_{x}^{-1} Q_{x} \otimes I_{y}\right)\left[\operatorname{diag}(\boldsymbol{\psi}) \operatorname{diag}\left(I_{x} \otimes P_{y}^{-1} Q_{y} \boldsymbol{\zeta}\right)\right] \mathbf{1}\right. \\
\left.-\left(I_{x} \otimes P_{y}^{-1} Q_{y}\right)\left[\operatorname{diag}(\boldsymbol{\psi}) \operatorname{diag}\left(P_{x}^{-1} Q_{x} \otimes I_{y} \boldsymbol{\zeta}\right)\right] \mathbf{1}\right\} \\
-\gamma \boldsymbol{\zeta}^{T}\left(P_{x} \otimes P_{y}\right)\left\{\left(I_{x} \otimes P_{y}^{-1} Q_{y}\right)\left[\operatorname{diag}(\boldsymbol{\zeta}) \operatorname{diag}\left(P_{x}^{-1} Q_{x} \otimes I_{y} \boldsymbol{\psi}\right)\right] \mathbf{1}\right. \\
\left.-\left(P_{x}^{-1} Q_{x} \otimes I_{y}\right)\left[\operatorname{diag}(\boldsymbol{\zeta}) \operatorname{diag}\left(I_{x} \otimes P_{y}^{-1} Q_{y} \boldsymbol{\psi}\right)\right] \mathbf{1}\right\} .
\end{array}
$$

In the first term on the RHS of (34) (the whole part multiplied by the $\alpha$ ) we use (22) and the commutative property of diagonal matrices to write

$$
\boldsymbol{\zeta}^{T}\left(P_{x} \otimes P_{y}\right)=\mathbf{1}^{T} \operatorname{diag}(\boldsymbol{\zeta})\left(P_{x} \otimes P_{y}\right)=\mathbf{1}^{T}\left(P_{x} \otimes P_{y}\right) \operatorname{diag}(\boldsymbol{\zeta}) .
$$

In the second and third term (the parts multiplied by $\beta$ and $\gamma$, respectively) we use $Q_{x, y}=-Q_{x, y}^{T},(22),(23)$ and (24) as follows

$$
\begin{aligned}
\zeta^{T}\left(P_{x} \otimes P_{y}\right)\left(P_{x}^{-1} Q_{x} \otimes I_{y}\right) & =\boldsymbol{\zeta}^{T}\left(Q_{x} \otimes P_{y}\right) \\
& =-\boldsymbol{\zeta}^{T}\left(Q_{x}^{T} P_{x}^{-1} \otimes I_{y}\right)\left(P_{x} \otimes P_{y}\right) \\
& =-\left[\left(P_{x} \otimes P_{y}\right)\left(P_{x}^{-1} Q_{x} \otimes I_{y}\right) \zeta\right]^{T} \\
& =-\mathbf{1}^{T} \operatorname{diag}\left[\left(P_{x} \otimes P_{y}\right)\left(P_{x}^{-1} Q_{x} \otimes I_{y}\right) \boldsymbol{\zeta}\right] \\
& =-\mathbf{1}^{T}\left(P_{x} \otimes P_{y}\right) \operatorname{diag}\left(P_{x}^{-1} Q_{x} \otimes I_{y} \boldsymbol{\zeta}\right)
\end{aligned}
$$




$$
\begin{aligned}
\boldsymbol{\zeta}^{T}\left(P_{x} \otimes P_{y}\right)\left(I_{x} \otimes P_{y}^{-1} Q_{y}\right) & =\boldsymbol{\zeta}^{T}\left(P_{x} \otimes Q_{y}\right) \\
& =-\boldsymbol{\zeta}^{T}\left(I_{x} \otimes Q_{y}^{T} P_{y}^{-1}\right)\left(P_{x} \otimes P_{y}\right) \\
& =-\left[\left(P_{x} \otimes P_{y}\right)\left(I_{x} \otimes P_{y}^{-1} Q_{y}\right) \zeta\right]^{T} \\
& =-\mathbf{1}^{T} \operatorname{diag}\left[\left(P_{x} \otimes P_{y}\right)\left(I_{x} \otimes P_{y}^{-1} Q_{y}\right) \boldsymbol{\zeta}\right] \\
& =-\mathbf{1}^{T}\left(P_{x} \otimes P_{y}\right) \operatorname{diag}\left(I_{x} \otimes P_{y}^{-1} Q_{y} \zeta\right) .
\end{aligned}
$$

Next, by extracting $\operatorname{diag}(\boldsymbol{\psi})$ and $\operatorname{diag}(\boldsymbol{\zeta})$ from the second and third term respectively, (34) becomes

$$
\begin{aligned}
\frac{1}{2} \frac{\partial}{\partial t}\|\boldsymbol{\zeta}\|_{\left(P_{x} \otimes P_{y}\right)}^{2}= & -\alpha \mathbf{1}^{T}\left(P_{x} \otimes P_{y}\right) \operatorname{diag}(\boldsymbol{\zeta}) \\
& \left\{\left[\operatorname{diag}\left(P_{x}^{-1} Q_{x} \otimes I_{y} \boldsymbol{\psi}\right) \operatorname{diag}\left(I_{x} \otimes P_{y}^{-1} Q_{y} \boldsymbol{\zeta}\right)\right] \mathbf{1}\right. \\
& \left.-\left[\operatorname{diag}\left(I_{x} \otimes P_{y}^{-1} Q_{y} \boldsymbol{\psi}\right) \operatorname{diag}\left(P_{x}^{-1} Q_{x} \otimes I_{y} \boldsymbol{\zeta}\right)\right] \mathbf{1}\right\} \\
& +\beta \mathbf{1}^{T}\left(P_{x} \otimes P_{y}\right) \operatorname{diag}(\boldsymbol{\psi}) \\
& \left\{-\operatorname{diag}\left(P_{x}^{-1} Q_{x} \otimes I_{y} \boldsymbol{\zeta}\right)\left[\operatorname{diag}\left(I_{x} \otimes P_{y}^{-1} Q_{y} \boldsymbol{\zeta}\right)\right] \mathbf{1}\right. \\
& \left.+\operatorname{diag}\left(I_{x} \otimes P_{y}^{-1} Q_{y} \boldsymbol{\zeta}\right)\left[\operatorname{diag}\left(P_{x}^{-1} Q_{x} \otimes I_{y} \boldsymbol{\zeta}\right)\right] \mathbf{1}\right\} \\
& +\gamma \mathbf{1}^{T}\left(P_{x} \otimes P_{y}\right) \operatorname{diag}(\boldsymbol{\zeta}) \\
& \left\{\operatorname{diag}\left(I_{x} \otimes P_{y}^{-1} Q_{y} \boldsymbol{\zeta}\right)\left[\operatorname{diag}\left(P_{x}^{-1} Q_{x} \otimes I_{y} \boldsymbol{\psi}\right)\right] \mathbf{1}\right. \\
& \left.-\operatorname{diag}\left(P_{x}^{-1} Q_{x} \otimes I_{y} \boldsymbol{\zeta}\right)\left[\operatorname{diag}\left(I_{x} \otimes P_{y}^{-1} Q_{y} \boldsymbol{\psi}\right)\right] \mathbf{1}\right\}
\end{aligned}
$$

Note that, thanks to the commutative property of diagonal matrices, the second term on the RHS of (37) is zero. Due to the same property, the first and the second term are the same with opposite sign if and only if

$$
\alpha=\gamma
$$

Finally, by canceling all the terms with opposite signs, we have

$$
\frac{1}{2} \frac{\partial}{\partial t}\|\zeta\|_{\left(P_{x} \otimes P_{y}\right)}^{2}=0 .
$$

This proves that $\mathbf{J}$ conserves enstrophy if and only if (38) is satisfied.

2. Conservation of kinetic energy

We consider (29) with the choice (38) and multiply (33) by $\boldsymbol{\psi}^{T}\left(P_{x} \otimes P_{y}\right)$. 
We obtain

$$
\begin{array}{r}
\boldsymbol{\psi}^{T}\left(P_{x} \otimes P_{y}\right) \frac{\partial \boldsymbol{\zeta}}{\partial t}=\frac{1}{2} \frac{\partial}{\partial t}\left\|(\nabla \boldsymbol{\psi})^{2}\right\|_{\left(P_{x} \otimes P_{y}\right)}^{2} \\
=-\alpha \boldsymbol{\psi}^{T}\left(P_{x} \otimes P_{y}\right)\left\{\left[\operatorname{diag}\left(P_{x}^{-1} Q_{x} \otimes I_{y} \boldsymbol{\psi}\right) \operatorname{diag}\left(I_{x} \otimes P_{y}^{-1} Q_{y} \boldsymbol{\zeta}\right)\right] \mathbf{1}\right. \\
\left.-\left[\operatorname{diag}\left(I_{x} \otimes P_{y}^{-1} Q_{y} \boldsymbol{\psi}\right) \operatorname{diag}\left(P_{x}^{-1} Q_{x} \otimes I_{y} \boldsymbol{\zeta}\right)\right] \mathbf{1}\right\} \\
-\beta \boldsymbol{\psi}^{T}\left(P_{x} \otimes P_{y}\right)\left\{\left(P_{x}^{-1} Q_{x} \otimes I_{y}\right)\left[\operatorname{diag}(\boldsymbol{\psi}) \operatorname{diag}\left(I_{x} \otimes P_{y}^{-1} Q_{y} \boldsymbol{\zeta}\right)\right] \mathbf{1}\right. \\
\left.-\left(I_{x} \otimes P_{y}^{-1} Q_{y}\right)\left[\operatorname{diag}(\boldsymbol{\psi}) \operatorname{diag}\left(P_{x}^{-1} Q_{x} \otimes I_{y} \boldsymbol{\zeta}\right)\right] \mathbf{1}\right\} \\
-\alpha \boldsymbol{\psi}^{T}\left(P_{x} \otimes P_{y}\right)\left\{\left(I_{x} \otimes P_{y}^{-1} Q_{y}\right)\left[\operatorname{diag}(\boldsymbol{\zeta}) \operatorname{diag}\left(P_{x}^{-1} Q_{x} \otimes I_{y} \boldsymbol{\psi}\right)\right] \mathbf{1}\right. \\
\left.-\left(P_{x}^{-1} Q_{x} \otimes I_{y}\right)\left[\operatorname{diag}(\boldsymbol{\zeta}) \operatorname{diag}\left(I_{x} \otimes P_{y}^{-1} Q_{y} \boldsymbol{\psi}\right)\right] \mathbf{1}\right\} .
\end{array}
$$

Similar to the procedure above for the first term on the RHS of (39) we rewrite

$$
\boldsymbol{\psi}^{T}\left(P_{x} \otimes P_{y}\right)=\mathbf{1}^{T} \operatorname{diag}(\boldsymbol{\psi})\left(P_{x} \otimes P_{y}\right)=\mathbf{1}^{T}\left(P_{x} \otimes P_{y}\right) \operatorname{diag}(\boldsymbol{\psi})
$$

In the second and third term we again apply $Q_{x, y}=-Q_{x, y}^{T},(23)$ and (24) obtaining, with analogous steps to (35) and (36),

$$
\boldsymbol{\psi}^{T}\left(P_{x} \otimes P_{y}\right)\left(P_{x}^{-1} Q_{x} \otimes I_{y}\right)=-\mathbf{1}^{T}\left(P_{x} \otimes P_{y}\right) \operatorname{diag}\left(P_{x}^{-1} Q_{x} \otimes I_{y} \boldsymbol{\psi}\right)
$$

and

$$
\boldsymbol{\psi}^{T}\left(P_{x} \otimes P_{y}\right)\left(I_{x} \otimes P_{y}^{-1} Q_{y}\right)=-\mathbf{1}^{T}\left(P_{x} \otimes P_{y}\right) \operatorname{diag}\left(I_{x} \otimes P_{y}^{-1} Q_{y} \boldsymbol{\psi}\right) .
$$

By extracting $\operatorname{diag}(\boldsymbol{\psi})$ and $\operatorname{diag}(\boldsymbol{\zeta})$ from the second and the third term, 
respectively, (39) becomes

$$
\begin{aligned}
\frac{\partial}{\partial t}\|(\nabla \boldsymbol{\psi})\|_{\left(P_{x} \otimes P_{y}\right)}^{2}= & -\alpha \mathbf{1}^{T}\left(P_{x} \otimes P_{y}\right) \operatorname{diag}(\boldsymbol{\psi}) \\
& \left\{\left[\operatorname{diag}\left(P_{x}^{-1} Q_{x} \otimes I_{y} \boldsymbol{\psi}\right) \operatorname{diag}\left(I_{x} \otimes P_{y}^{-1} Q_{y} \boldsymbol{\zeta}\right)\right] \mathbf{1}\right. \\
& \left.-\left[\operatorname{diag}\left(I_{x} \otimes P_{y}^{-1} Q_{y} \boldsymbol{\psi}\right) \operatorname{diag}\left(P_{x}^{-1} Q_{x} \otimes I_{y} \boldsymbol{\zeta}\right)\right] \mathbf{1}\right\} \\
& +\beta \mathbf{1}^{T}\left(P_{x} \otimes P_{y}\right) \operatorname{diag}(\boldsymbol{\psi}) \\
& \left\{\operatorname{diag}\left(P_{x}^{-1} Q_{x} \otimes I_{y} \boldsymbol{\psi}\right)\left[\operatorname{diag}\left(I_{x} \otimes P_{y}^{-1} Q_{y} \boldsymbol{\zeta}\right)\right] \mathbf{1}\right. \\
& \left.-\operatorname{diag}\left(I_{x} \otimes P_{y}^{-1} Q_{y} \boldsymbol{\psi}\right)\left[\operatorname{diag}\left(P_{x}^{-1} Q_{x} \otimes I_{y} \boldsymbol{\zeta}\right)\right] \mathbf{1}\right\} \\
& +\alpha \mathbf{1}^{T}\left(P_{x} \otimes P_{y}\right) \operatorname{diag}(\boldsymbol{\zeta}) \\
& \left\{-\operatorname{diag}\left(I_{x} \otimes P_{y}^{-1} Q_{y} \boldsymbol{\psi}\right)\left[\operatorname{diag}\left(P_{x}^{-1} Q_{x} \otimes I_{y} \boldsymbol{\psi}\right)\right] \mathbf{1}\right. \\
& \left.+\operatorname{diag}\left(P_{x}^{-1} Q_{x} \otimes I_{y} \boldsymbol{\psi}\right)\left[\operatorname{diag}\left(I_{x} \otimes P_{y}^{-1} Q_{y} \boldsymbol{\psi}\right)\right] \mathbf{1}\right\}
\end{aligned}
$$

Note again that, thanks to the commutative property of diagonal matrices, the last term on the RHS of eq. (40) is zero. While the second and third term are the same with opposite sign if and only if

$$
\alpha=\beta \text {. }
$$

By summation, we have

$$
\frac{1}{2} \frac{\partial}{\partial t}\|(\nabla \boldsymbol{\psi})\|_{\left(P_{x} \otimes P_{y}\right)}^{2}=0
$$

Hence, J conserves kinetic energy only assuming (41).

3. Skew-symmetry

We conclude noting that $\mathbf{J}_{1}$ is itself skew-symmetric. By definition (26):

$$
\mathbf{J}_{1}(a, b)=-\mathbf{J}_{1}(b, a),
$$

while $\mathbf{J}_{2}$ and $\mathbf{J}_{3}$ are not skew-symmetric. However, from definitions (27)-(28) it follows that they satisfy

$$
\mathbf{J}_{2}(a, b)=-\mathbf{J}_{3}(b, a) .
$$


Thus, the linear combination $\mathbf{J}$ is antisymmetric if $\beta=\gamma$, since in that case we have

$$
\begin{aligned}
\mathbf{J}(a, b) & =\alpha \mathbf{J}_{1}(a, b)+\beta \mathbf{J}_{2}(a, b)+\beta \mathbf{J}_{3}(a, b) \\
& =-\alpha \mathbf{J}_{1}(b, a)-\beta \mathbf{J}_{3}(b, a)-\beta \mathbf{J}_{2}(b, a) \\
& =-\mathbf{J}(b, a) .
\end{aligned}
$$

In conclusion, $\mathbf{J}$ conserves enstrophy, kinetic energy and it is skew-symmetric at the same time, if and if only we choose $\alpha=\beta=\gamma$. This condition, together with (30), gives exactly (31) and $\mathbf{J}$ becomes $\mathbf{J}^{*}$.

$\mathbf{J}^{*}$ in (32) is an Arakawa-like arbitrary high-order finite difference approximation of the non-linear Jacobian in (19).

\section{Numerical Results}

\subsection{Accuracy}

In this section we show the rate of convergence of the approximation (32) using SBP operators with different orders of accuracy [26]. We use the method of manufactured solution and choose the analytical stream function

$$
\psi(x, y, t)=K[\sin (2 \pi(a x-t)+\cos (2 \pi(b y-t)],
$$

related to the vorticity function

$$
\zeta=\Delta \psi=-4 \pi^{2} K\left[a ^ { 2 } \operatorname { s i n } \left(2 \pi(a x-t)+b^{2} \cos (2 \pi(b y-t)] .\right.\right.
$$

Here $K$ is a constant chosen such that $\max |\boldsymbol{\zeta}|=1$, while $a, b$ determine the frequencies in the $x$ and $y$ direction respectively. The functions (43) and (44) satisfy the equation

$$
\frac{\partial \zeta}{\partial t}+J(\psi, \zeta)=F
$$

where $F$ is the forcing term obtained by inserting (43) and (44) into (8).

A full time-space discretization of (45) is obtained by combining the scheme (32) with a suitable high order time integration scheme. For all the tests in this paper we have used the explicit standard fourth-order RungeKutta (RK4) scheme with time step $\Delta t$ obtained from the relation $\Delta t=$

$C(h)^{p / 4}$, where $p$ is the order the accuracy in space and $h$ is the spatial step (the same in the $x$ and $y$ direction). $C$ is a scaling factor for keeping the error in time negligible with respect to the error in space. 
The convergence rate is calculated using

$$
p=\log \left(\frac{\left\|\zeta-\zeta^{h_{1}}\right\|_{2}}{\left\|\zeta-\zeta^{h_{2}}\right\|_{2}}\right) \backslash \log \left(\frac{h_{1}}{h_{2}}\right),
$$

where $\zeta$ is the analytical solution, $\zeta^{h}$ the corresponding numerical solution with spatial step $h$ and $\left\|\zeta-\zeta^{h}\right\|_{2}$ is the discrete $L^{2}$ norm. We consider SBP operators with 2nd, 4th, 6th and 8th expected order of accuracy [26]. The results at the final time $T=0.1$ are presented in Table 5.1. In all the the computations we have chosen the frequencies $a=2$ and $b=3$ in the $x$ and $y$ direction, respectively, and $C=1 / 50$ for rescaling the time step. All the schemes show the design order of accuracy.

\begin{tabular}{l|ll|ll|ll|lll}
\hline & SBP 2nd & & SBP 4th & & SBP 6th & & SBP 8th & \\
$\mathrm{N}$ & Err & $p$ & Err & $p$ & Err & $p$ & Err & $p$ \\
\hline 30 & $2.1110^{-2}$ & - & $6.6610^{-4}$ & - & $1.0010^{-4}$ & - & $8.1310^{-6}$ & - \\
40 & $1.1010^{-2}$ & 1.97 & $4.3310^{-4}$ & 3.95 & $1.8410^{-5}$ & 5.88 & $8.5210^{-7}$ & 7.83 \\
50 & $7.5010^{-3}$ & 1.94 & $1.7910^{-4}$ & 3.94 & $4.9010^{-6}$ & 5.93 & $1.4610^{-7}$ & 7.90 \\
60 & $5.3410^{-3}$ & 2.03 & $8.6710^{-5}$ & 3.99 & $1.6510^{-6}$ & 5.95 & $3.4310^{-8}$ & 7.93 \\
70 & $3.9210^{-3}$ & 2.00 & $4.6910^{-5}$ & 3.98 & $6.5910^{-7}$ & 5.96 & $1.0010^{-8}$ & 7.95 \\
80 & $1.9710^{-3}$ & 1.97 & $2.7510^{-5}$ & 3.98 & $5.9710^{-7}$ & 5.97 & $3.4810^{-9}$ & 7.96 \\
\hline
\end{tabular}

Table 1: Discrete $L^{2}$-error and Rate of convergence for the 2nd, 4th, 6th and 8th SBP operators. The error is indicated with $E r r$ and the order of accuracy with $p . \mathrm{N}$ indicates the number of grid points in the $x$ and $y$ direction, respectively.

\subsection{Efficiency}

We compare the Arakawa Jacobian (or second order version of (32)) and the other higher order schemes derived in this paper. The manufactured solutions (43) and (44) are used as before. As efficiency parameters we consider the number of grid points and the CPU time (in seconds) needed to reach the magnitude of the error. Table 5.2 shows the efficiency results with a reference error of magnitude less than $0.5 \cdot 10^{-4}$. All the computations are done with a fixed time step $\Delta t=10^{-3}$, advancing up to the final time $T=0.1$. Clearly, the high order SBP schemes are much more efficient than the Arakawa approximation. 


\begin{tabular}{l|l|l|l|l}
\hline & Arakawa & SBP 4th & SBP 6th & SBP 8th \\
\hline Error & $4.83 \cdot 10^{-4}$ & $4.34 \cdot 10^{-4}$ & $4.70 \cdot 10^{-4}$ & $4.08 \cdot 10^{-4}$ \\
$\mathrm{CPU}$ & $1215.614 \mathrm{~s}$ & $2.091 \mathrm{~s}$ & $0.287 \mathrm{~s}$ & $0.139 \mathrm{~s}$ \\
$\mathrm{~N}$ & 200 & 40 & 23 & 18 \\
\hline
\end{tabular}

Table 2: Efficiency comparison between the Arakawa (or second order SBP) scheme and high order approximations of (32) using SBP operators 4th, 6th and 8th order. The CPU time (in seconds) and the number of grid points $(\mathrm{N})$ are considered at the reference error of magnitude $10^{-4}$.

\section{Summary and Conclusions}

We have derived the two-dimensional vorticity equation from the incompressible inviscid Euler equation and showed that it was well-posed for periodic problems. We have focused on the advection term in the equation which can be rewritten as a Jacobian operator.

An Arakawa-like scheme for the discretization of the Jacobian has been formulated using a finite difference method based on summation-by-parts operators and splitting techniques. The resulting Jacobian approximation is mimetic, skew-symmetric, energy and enstrophy conserving and high-order accurate.

The skew-symmetry and the conservation properties have been proved by using the special structure of the SBP operators and a special way to relate vectors and diagonal matrices.

The numerical results show that the new Arakawa-like schemes using periodic SBP operators are high order accurate and more efficient than the original one presented in [3].

\section{Appendix A. The Arakawa scheme and the second order SBP formulation}

Here we compare the Arakawa's scheme derived in [3] with the discretization (32) using the second order SBP operator in [26]. We want to show that the second order approximation (32) at an internal grid point is identical to the one in [3]. 
The Arakawa's Jacobian at the grid point $\left(x_{i}, y_{j}\right), i, j=1, \ldots, N$, is

$$
\begin{aligned}
\mathbf{J}_{i, j}^{A}= & \frac{1}{12 h^{2}}\left[\left(\zeta_{i, j+1}-\zeta_{i, j-1}\right)\left(\psi_{i+1, j}-\psi_{i-1, j}\right)\right. \\
& \left.-\left(\zeta_{i+1, j}-\zeta_{i-1, j}\right)\left(\psi_{i, j+1}-\psi_{i, j-1}\right)\right] \\
& +\frac{1}{12 h^{2}}\left[\left(\zeta_{i+1, j+1}-\zeta_{i+1, j-1}\right) \psi_{i+1, j}-\left(\zeta_{i-1, j+1}-\zeta_{i-1, j-1}\right) \psi_{i-1, j}\right. \\
& \left.-\left(\zeta_{i+1, j+1}-\zeta_{i-1, j+1}\right) \psi_{i, j+1}+\left(\zeta_{i+1, j-1}-\zeta_{i-1, j-1}\right) \psi_{i, j-1}\right] \\
& -\frac{1}{12 h^{2}}\left[\left(\psi_{i+1, j+1}-\psi_{i+1, j-1}\right) \zeta_{i+1, j}-\left(\psi_{i-1, j+1}-\psi_{i-1, j-1}\right) \zeta_{i-1, j}\right. \\
& \left.+\left(\psi_{i+1, j+1}-\psi_{i-1, j+1}\right) \zeta_{i, j+1}-\left(\psi_{i+1, j-1}-\psi_{i-1, j-1}\right) \zeta_{i, j-1}\right] .
\end{aligned}
$$

The second order periodic operator on SBP form is

$$
P^{-1} Q=\left[\begin{array}{ccccc}
0 & \frac{1}{2 h} & & & -\frac{1}{2 h} \\
-\frac{1}{2 h} & 0 & \frac{1}{2 h} & & \\
& \ddots & \ddots & \ddots & \\
& & -\frac{1}{2 h} & 0 & \frac{1}{2 h} \\
\frac{1}{2 h} & & & -\frac{1}{2 h} & 0
\end{array}\right],
$$

where $h$ is the spatial step. Hence, the second order partial derivatives (25) of the vector function $\mathbf{f}=\left(f_{11}, \ldots, f_{1 M}, f_{21} \ldots, f_{2 M}, \ldots, f_{N 1}, \ldots, f_{N M}\right)^{T}$ are

$$
\left(P_{x}^{-1} Q_{x} \otimes I_{y}\right) \mathbf{f}=\left[\begin{array}{ccccc}
\ddots & \ddots & \ddots & & \\
& -\frac{1}{2 \Delta_{x}} I_{y} & 0 & \frac{1}{2 \Delta_{x}} I_{y} & \\
& & \ddots & \ddots & \ddots
\end{array}\right] \cdot\left[\begin{array}{c}
\vdots \\
f_{i, j} \\
\vdots
\end{array}\right]=\left[\begin{array}{c}
\vdots \\
\frac{-f_{i-1, j}+f_{i+1, j}}{2 \Delta_{x}} \\
\vdots
\end{array}\right]
$$

and

$$
\left(I_{x} \otimes P_{y}^{-1} Q_{y}\right) \mathbf{f}=\left[\begin{array}{ccccc}
\ddots & \ddots & \ddots & & \\
& 0 & P_{y}^{-1} Q_{y} & 0 & \\
& & \ddots & \ddots & \ddots
\end{array}\right] \cdot\left[\begin{array}{c}
\vdots \\
f_{i, j} \\
\vdots
\end{array}\right]=\left[\begin{array}{c}
\vdots \\
\frac{-f_{i, j-1}+f_{i, j+1}}{2 \Delta_{y}} \\
\vdots
\end{array}\right] .
$$


Choosing $\Delta_{x}=\Delta_{y}=h$ in (49) and (50) lets us rewrite $\mathbf{J}_{1}(\psi, \zeta)$ as

$$
\begin{aligned}
\mathbf{J}_{1}(\boldsymbol{\psi}, \boldsymbol{\zeta}) & =\operatorname{diag}\left(. ., \frac{\left(-\psi_{i-1, j}+\psi_{i+1, j}\right)}{2 h}, . .\right) \cdot \operatorname{diag}\left(. ., \frac{\left(-\zeta_{i, j-1}+\zeta_{i, j+1}\right)}{2 h}, . .\right) \mathbf{1} \\
& -\operatorname{diag}\left(. ., \frac{\left(-\psi_{i, j-1}+\psi_{i, j+1}\right)}{2 h}, . .\right) \cdot \operatorname{diag}\left(. ., \frac{\left(-\zeta_{i-1, j}+\zeta_{i+1, j}\right)}{2 h}, . .\right) \mathbf{1} .
\end{aligned}
$$

Hence, $\mathbf{J}_{1}(\boldsymbol{\psi}, \boldsymbol{\zeta})$ at the grid point $\left(x_{i}, y_{j}\right)$ is given by

$$
\mathbf{J}_{1_{i, j}}=\frac{1}{4 h^{2}}\left[\left(\psi_{i+1, j}-\psi_{i-1, j}\right)\left(\zeta_{i, j+1}-\zeta_{i, j-1}\right)-\left(\psi_{i, j+1}-\psi_{i, j-1}\right)\left(\zeta_{i+1, j}-\zeta_{i-1, j}\right)\right] \text {. }
$$

In a similar way we compute $\mathbf{J}_{2}(\psi, \zeta)$ :

$$
\left.\begin{array}{l}
\mathbf{J}_{2}(\boldsymbol{\psi}, \boldsymbol{\zeta})=\left(P_{x}^{-1} Q_{x} \otimes I_{y}\right)\left[\operatorname{diag}(\boldsymbol{\psi}) \cdot \operatorname{diag}\left(. ., \frac{\left(-\zeta_{i, j-1}+\zeta_{i, j+1}\right)}{2 h}, . .\right) \mathbf{1}\right] \\
-\left(I_{x} \otimes P_{y}^{-1} Q_{y}\right)\left[\operatorname{diag}(\boldsymbol{\psi}) \cdot \operatorname{diag}\left(. ., \frac{\left(-\zeta_{i-1, j}+\zeta_{i+1, j}\right)}{2 h}, . .\right) \mathbf{1}\right] \\
=\left(P_{x}^{-1} Q_{x} \otimes I_{y}\right)\left[\frac{\psi_{i, j}\left(-\zeta_{i, j-1}+\zeta_{i, j+1}\right)}{2 h}\right]-\left(I_{x} \otimes P_{y}^{-1} Q_{y}\right)\left[\frac{\psi_{i, j}\left(-\zeta_{i-1, j}+\zeta_{i+1, j}\right)}{2 h}\right. \\
\vdots
\end{array}\right]
$$

which gives $\mathbf{J}_{2}(\psi, \zeta)$ at the grid point $\left(x_{i}, y_{j}\right)$ as

$$
\begin{aligned}
\mathbf{J}_{2_{i, j}} & =\frac{1}{4 h^{2}}\left[\psi_{i+1, j}\left(\zeta_{i+1, j+1}-\zeta_{i+1, j-1}\right)-\psi_{i-1, j}\left(\zeta_{i-1, j+1}-\zeta_{i-1, j-1}\right)\right. \\
& \left.+\psi_{i, j+1}\left(\zeta_{i+1, j+1}-\zeta_{i-1, j+1}\right)-\psi_{i, j-1}\left(\zeta_{i+1, j-1}-\zeta_{i-1, j-1}\right)\right] .
\end{aligned}
$$

For $\mathbf{J}_{3}(\psi, \zeta)$ we obtain

$$
\left.\begin{array}{l}
\mathbf{J}_{3}(\boldsymbol{\psi}, \boldsymbol{\zeta})=-\left(P_{x}^{-1} Q_{x} \otimes I_{y}\right)\left[\operatorname{diag}(\boldsymbol{\zeta}) \cdot \operatorname{diag}\left(. ., \frac{\left(-\psi_{i, j-1}+\psi_{i, j+1}\right)}{2 h}, . .\right) \mathbf{1}\right] \\
+\left(I_{x} \otimes P_{y}^{-1} Q_{y}\right)\left[\operatorname{diag}(\boldsymbol{\zeta}) \cdot \operatorname{diag}\left(. ., \frac{\left(-\psi_{i-1, j}+\psi_{i+1, j}\right)}{2 h}, . .\right) \mathbf{1}\right] \\
=-\left(P_{x}^{-1} Q_{x} \otimes I_{y}\right)\left[\frac{\zeta_{i, j}\left(-\psi_{i, j-1}+\psi_{i, j+1}\right)}{2 h}\right]+\left(I_{x} \otimes P_{y}^{-1} Q_{y}\right)\left[\frac{\zeta_{i, j}\left(-\psi_{i-1, j}+\psi_{i+1, j}\right)}{2 h}\right. \\
\vdots
\end{array}\right]
$$


Finally, $\mathbf{J}_{3}(\psi, \zeta)$ at the grid point $\left(x_{i}, y_{j}\right)$ is

$$
\begin{aligned}
\mathbf{J}_{3_{i, j}} & =\frac{1}{4 h^{2}}\left[\zeta_{i-1, j}\left(\psi_{i-1, j+1}-\psi_{i-1, j-1}\right)-\zeta_{i+1, j}\left(\psi_{i+1, j+1}-\psi_{i+1, j-1}\right)\right. \\
& \left.+\zeta_{i, j-1}\left(\psi_{i+1, j-1}-\psi_{i-1, j-1}\right)-\zeta_{i, j+1}\left(\psi_{i+1, j+1}-\psi_{i-1, j+1}\right)\right] .
\end{aligned}
$$

The average of (51),(52) and (53) gives the expression for $\mathbf{J}^{*}(\boldsymbol{\psi}, \boldsymbol{\zeta})$ at the grid point $\left(x_{i}, y_{j}\right)$ as

$$
\begin{aligned}
\mathbf{J}_{i, j}^{*}= & \frac{1}{12 h^{2}}\left[\left(\psi_{i+1, j}-\psi_{i-1, j}\right)\left(\zeta_{i, j+1}-\zeta_{i, j-1}\right)-\left(\psi_{i, j+1}-\psi_{i, j-1}\right)\left(\zeta_{i+1, j}-\zeta_{i-1, j}\right)\right] \\
+ & \frac{1}{12 h^{2}}\left[\psi_{i+1, j}\left(\zeta_{i+1, j+1}-\zeta_{i+1, j-1}\right)-\psi_{i-1, j}\left(\zeta_{i-1, j+1}-\zeta_{i-1, j-1}\right)\right. \\
& \left.\quad+\psi_{i, j+1}\left(\zeta_{i+1, j+1}-\zeta_{i-1, j+1}\right)-\psi_{i, j-1}\left(\zeta_{i+1, j-1}-\zeta_{i-1, j-1}\right)\right] \\
+ & \frac{1}{12 h^{2}}\left[\zeta_{i-1, j}\left(\psi_{i-1, j+1}-\psi_{i-1, j-1}\right)-\zeta_{i+1, j}\left(\psi_{i+1, j+1}-\psi_{i+1, j-1}\right)\right. \\
& \left.\zeta_{i, j-1}\left(\psi_{i+1, j-1}-\psi_{i-1, j-1}\right)-\zeta_{i, j+1}\left(\psi_{i+1, j+1}-\psi_{i-1, j+1}\right)\right] .
\end{aligned}
$$

The expressions (47) and (54) are identical. Thus, the Arakawa's Jacobian coincides with the classical second order SBP formulation (32).

\section{References}

[1] Antonella Abbà, Luca Buonaventura. A mimetic finite difference discretization for the incompressible Navier-Stokes equations, International Journal for Numerical Methods in Fluids, Vol. 56, No. 8, pp: 1101-1106, March (2008)

[2] Rafail V. Abramov and Andrew J. Majda Discrete Approximations with Additional Conserved Quantities: Deterministic and Statistical Behavior Methods Appl. Anal., Vol. 10, No. 2, pp:151-190 (2003)

[3] Akio Arakawa Computational Design for Long-Term Numerical Integration of the Equations of Fluid Motion: Two-Dimensional Incompressible Flow. Part I Journal of Computational Physics, 1, pp:119-143 (1966)

[4] Akio Arakawa Finite-difference Methods in Climate Modeling Physically-Based Modeling and Simulation of Climate and Climatic Change, 243, pp:79-168 (1988) 
[5] Batchelor, G. K. (1967) An Introduction to Fluid Dynamics, Cambridge University Press, ISBN 0-521-09817-3.

[6] Mark H. Carpenter, Jan Nordström, and David Gottlieb A Stable and Conservative Interface Treatment of Arbitrary Spatial Accuracy. Journal of Computational Physics, 148, pp:341-365 (1999)

[7] Dennis C Jespersen Arakawa's Method Is a Finite-Element Method Journal of Computational Physics, 16, pp:383-390 (1974)

[8] S. Dubinkina, J. Frank Statistical mechanics of Arakawa's discretization Annals of Physics, 227 (2), pp:1286-1305 (2007)

[9] Dale R. Durran. Numerical Methods for Fluid Dynamics With Applications to Geophysics Second edition. Springer (2010).

[10] B. Gustafsson, H.-O. Kreiss, J. Oliger. Time Dependent Problems and Difference Methods, John Wiley \& Sons, Inc., (1995).

[11] D.B. Haidvogel, I.M. Held. Homogeneous quasi-geostrophic turbulence driven by a uniform temperature gradient Journal of the Atmospheric Sciences 37 pp. 2644-2660 (1980).

[12] A. Kacimi, B. Khouider A numerical investigation of the barotropic instability on the equatorial $\beta$-plane Theoretical and Computational Fluid Dynamic, (2012)

[13] H.-O. Kreiss and Godela Scherer. On the existence of energy estimates for difference approximations for hyperbolic systems. Technical report, Uppsala University, Division of Scientific Computing, 1977.

[14] Bas van't Hof a, Arthur E.P. Veldman Mass, momentum and energy conserving (MaMEC) discretizations on general grids for the compressible Euler and shallow water equations Journal of Computational Physics 231, 4723-4744 (2012)

[15] W. R. Holland. The role of mesoscale eddies in the general circulation of the ocean: Numerical experiments using a wind-driven quasi-geostrophic model J. Phys. Oceanogr. 8 pp.363-392 (1978).

[16] James R. Holton. An introduction to dynamic meteorology Fourth edition. Academic Press, 2004. 
[17] H. Lamb (1932), Hydrodynamics (6th ed.), Cambridge University Press, republished by Dover Publications, ISBN 0-486-60256-7.

[18] D. Lilly On the computational stability of numerical solutions of time dependent non-linear geophysical fluid dynamics problems Geophysical Fluid Dynamics Laboratory, U.S. Weather Bureau, Washington, D.C. 93 (1) pp: 11-26 (1965)

[19] Robert I. McLachlan Spatial discretization of partial differential equations with integrals Journal of Numerical Analysis, 23, n.4, pp:645-664 (2003)

[20] Andrew T. T. McRae, and Colin J. Cotter Energy- and enstrophy- conserving schemes for the shallow-water equations, based on mimetic finite elements Quarterly Journal of the Royal Meteorological Society, Vol. 140, N. 684, pp: 2223-2234, October (2014)

[21] Jan Nordström. Conservative Finite Difference Formulations, Variable Coefficients, Energy Estimates and Artificial Dissipation Journal of Scientific Computing, Vol. 29, No. 3, December (2006)

[22] Joseph Pedlosky. Geophysical Fluid Dynamics. Second edition. SpringerVerlag (1998).

[23] Norman A. Phillips An example of Non-Linear Computational Instability The Atmosphere and the Sea in Motion (Rockefeller Institute Press in association with Oxford University Press, New York), pp:501-504-38 (1959)

[24] Rick Salmon and Lynne D Talley Generalizations of Arakawa's Jacobian Journal of Computational Physics, 83, pp:247-259 (1989)

[25] O. San, A. E. Staples High-order methods for decaying two-dimensional homogeneous isotropic turbulence Computers Fluids, 53 pp:105-127 (2012)

[26] Bo Strand Summation by parts for finite difference approximations for $d / d x$, Journal of Computational Physics, 110, pp:47-67 (1994)

[27] Magnus Svärd, Jan Nordström Review of summation-by-parts schemes for initial-boundary-value problems, Journal of Computational Physics, 268, pp:17-38 (2014) 